\title{
PERSISTIR A TRAVÉS DEL CAMBIO: LA RECONVERSIÓN DE “LA ESCUELA-RESIDENCIA DE BELL-LLOC” (LA ROCA, BARCELONA, 1964)*
}

Amaya Martínez Marcos**

Grupo de investigación consolidado FORM

Universidad Politécnica de Cataluña, Barcelona, España

Recibido: I agosto 2013

Aprobado: 26 septiembre 2013
Vista desde la cubierta hacia la residencia y zona ampliada.

Fuente: Fotografía de Amaya M. M., 2011.

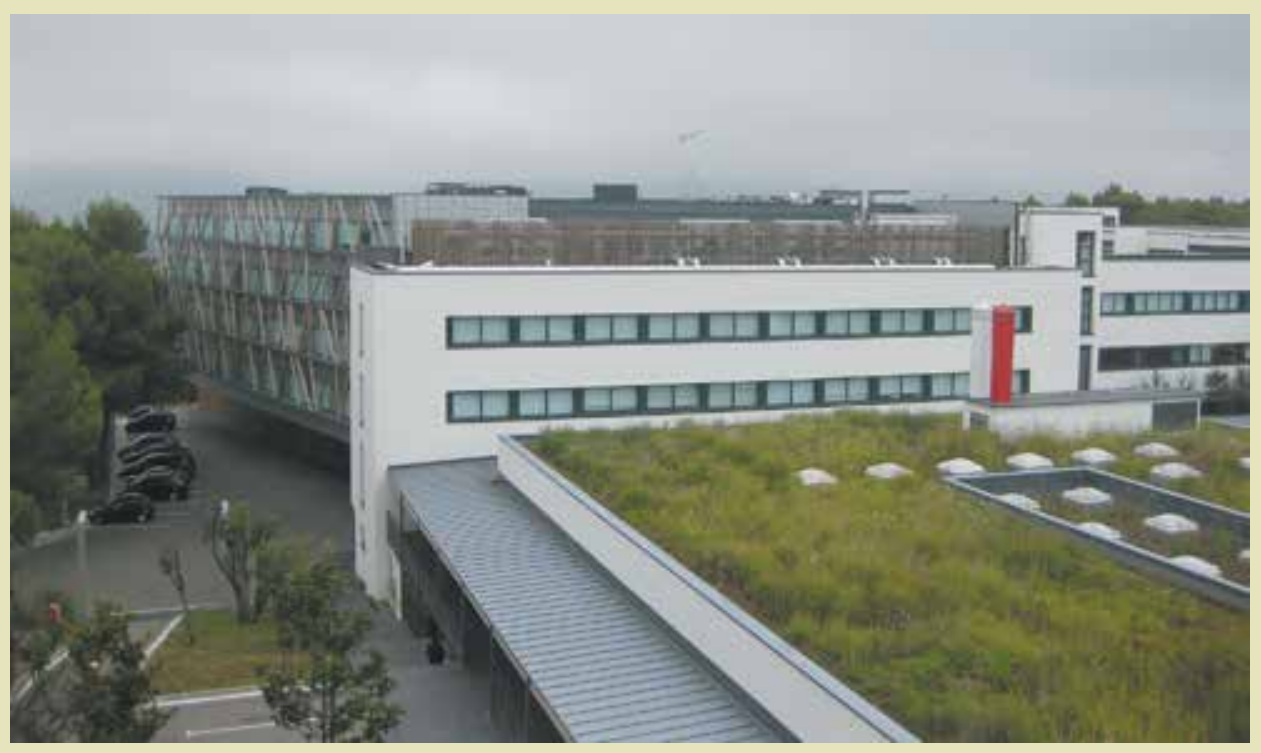

\section{RESUMEN}

El objetivo de este artículo es desvelar la permanencia y transformación del patrimonio moderno escolar español a través del caso de la "Escuela-Residencia de Bell-Lloc", situada en el municipio de La Roca (Barcelona). El conjunto escolar refleja el proceder de un momento en el que se generaron nuevas instituciones docentes ajenas al Estado. El arquitecto Manuel Baldrich i Tibau supo unificar los valores universales de la modernidad, junto a los propios del lugar y de la especificidad del programa, con el fin de atender aspectos de sostenibilidad en el conjunto escolar. La investigación se centra en el análisis de su concepción y diseño como procedimiento de reconocimiento e identificación de sus valores modernos. A través de los conceptos "preservación", "adecuación" y "ampliación" se muestra cuál es el proceso de transformación seguido hasta la actualidad. En 1994 la escuela-residencia quedó en desuso pero mantuvo su estado original. Recientemente, el conjunto ha sido transformado en un centro de negocios donde se han instalado diversas compañías. Todo ello con el objetivo de evaluar posibles criterios de intervención, extrapolable a otros casos de la modernidad en situación de abandono o desuso.

\section{PALABRAS CLAVE}

Arquitectura Escolar, Escuela, Patrimonio Moderno, Modernidad, Proyecto Arquitectónico, Escuela-Residencia De Bell-Lloc, Reconversión, Preservación, Permanencia, Transformación. 


\section{SURVIVE THROUGH CHANGE: THE CONVERSION OF "BELL-LLOC SCHOOL-RESIDENCE" (LA ROCA, BARCELONA, 1964)}

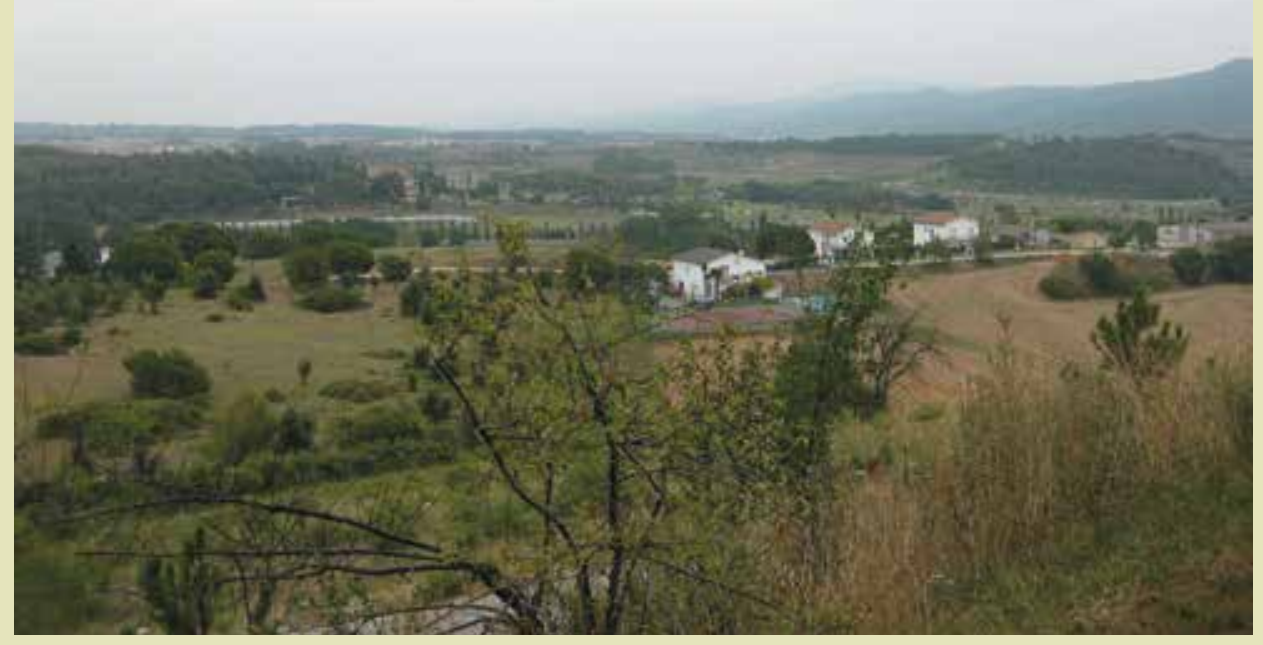

\section{ABSTRACT}

The aim of this work is to reveal the permanence and transformation of the modern Spanish school heritage through the case of "Bell-Lloc School-Residence" built in La Roca (Barcelona). It reflects the procedure of a time in which were created new educational institutions without the influence of the State. The architect Manuel Baldrich I Tibau was able to combine in it all the universal values of modernity. The consideration of the location and the program needs are also included in order to resolve aspects such as sustainability. The research focuses on the analysis of the origin and of its design as a process of recognition and identification of its modern values. Through the concepts of "preservation", "adequacy" and "extension" is revealed the "conversion" process followed to date. In 1994 the school-residence become in disused but keeping its original state. Recently the whole complex has been converted in a business centre in which several companies have settled. With the objective of evaluate possible intervention criteria that could be applied to other cases in a non-use situation.

\section{KEYWORDS}

School Buildings, Schools, Modern Heritage, Modernity, Architectural Design, Bell-Lloc School-Residence, Barcelona, Conversion, Preservation, Permanence, Transformation.
Vista desde la cubierta hacia la residencia y zona ampliada. Fuente: Amaya M. M., 20II. periodo 2008-20I I es Becaria de Investigación de la Generalitat de Catalunya y profesora en el Máster Universitario en "Teoría y Práctica del Proyecto de Arquitectura” UPC.

E:mail: amayamar@gmail.com 


\section{INTRODUCCIÓN}

El patrimonio moderno, su permanencia y transformación, constituye uno de los principales ejes de debate y controversia en el panorama de la arquitectura actual, y en particular en lo que se refiere a los posibles criterios de intervención sobre el mismo. En el reciente "Madrid Document for Twentieh-Century Architectural Heritage" (20I I), presentado por el ISC20 ' , se muestra la necesidad de generar un marco teórico para proteger y generar las pautas básicas que permitan intervenir sobre el patrimonio moderno.

Intervenir sobre el patrimonio de la modernidad implica nuevas consideraciones sobre la manera de preservar y actuar, debido a su proximidad temporal y matérica y se contrapone a la intervención sobre la arquitectura de corte histórico y cuyos criterios ya quedaron definidos a nivel internacional en la "Carta de Venecia" (1964). Estos principios, que básicamente son recuperados en "The Madrid Document" para su aplicación sobre el patrimonio moderno, y a los que se añade la consideración de intervención, o de incluso su modificación para ser revitalizados y continuar constituyendo no solo un patrimonio histórico sino un patrimonio vivo, con uso. No hay que olvidar que la Arquitectura Moderna se constituye de edificios emblemáticos ya reconocidos a nivel internacional, pero también de otros aportes novedosos, como la intensa investigación en el campo de la vivienda y la generación de nuevos usos acordes a las necesidades específicas de una época específica. Éste sería el caso de los equipamientos destinados a ser usados en las labores de la docencia, la sanidad o en la industria. Muchos de estos edificios ya han sido registrados, catalogados y protegidos por entidades como DOCOMOMO².

En términos generales, son los equipamientos modernos los que constituyen en sí una tipología definida capaz de albergar, hoy en día, nuevos y diferentes usos a través de la aplicación de conceptos "RE" como rehabitar, reconvertir, recuperar, refuncionalizar, rehabilitar, revitalizar, entre otros, bajo la idea de aprovechar las estructuras ya existentes en el territorio. En algunos casos, el uso queda obsoleto, pero no pasa lo mismo con su validez formal, estética o icónica del conjunto, reconociendo así que su estructura, incluso cuanto más precisa, más usos es capaz de albergar. Se defiende la intervención en aquellos casos en los que se encuentran en riesgo de pérdida o destrucción; pero manteniendo el significado cultural y las consideraciones originales de la construcción. Todo ello con la finalidad de dotar de vida al edificio y al lugar, a su huella dejada en el territorio por el paso del tiempo y que constituye parte de la memoria colectiva, bajo la pretensión de huir de la momificación del objeto arquitectónico en sí mismo. Estos aspectos adquieren significado a través de las palabras de Montaner (20II) "en unas sociedades en las que envejece su patrimonio y sus habitantes, los procesos de rehabilitación son la clave para que se fomente la proximidad y la superposición de usos y actividades, y se potencie un uso social del tiempo".

Bajo estas premisas, se analiza el origen, el proyecto y las estrategias de actuación llevadas a cabo en el conjunto escolar objeto de la investigación. El edificio de la Escuela-Residencia de Bell-lloc en La Roca del Vallès, obra del arquitecto Manuel Baldrich Tibau ${ }^{3}$, y realizado

I International Scientific Comittee on 20th Century Heritage (ICOMOS) dio a conocer The Madrid Document en la International Conference Intervention Approaches for the 20th Century Architectural Heritage que tuvo lugar los días 14, 15 y 16 de Junio de $20 \mathrm{I}$ I en Madrid. En la Conferencia Internacional se abordaron tres temas fundamentales sobre el patrimonio arquitectónico del siglo XX: su identificación y reconocimiento, un acercamiento a la conservación y protección y un acercamiento a la intervención.

2 DOCOMOMO, Documentation and Conservation of Building, Sites and Neighbourhoods of the Modern Movement, organización internacional creada en 1990 con el objetivo de inventariar, divulgar y proteger el patrimonio arquitectónico del Movimiento Moderno. La sección DOCOMOMO Ibérico coordina, desde su formación en 1993, los objetivos en España y Portugal.

3 Manuel Baldrich Tibau (1911-1966) se titula como Arquitecto en 1935. Desarrolló una gran actividad tanto en el ámbito urbanístico como en el arquitectónico, además, publicó libros y escritos, participó en debates, congresos, e impartió cursos y seminarios. Destacan entre otras obras Los Hogares Mundet (1954-1957), el Polígono de Montbau (196I-1965) o la Piscina Sant Jordi (1966). 


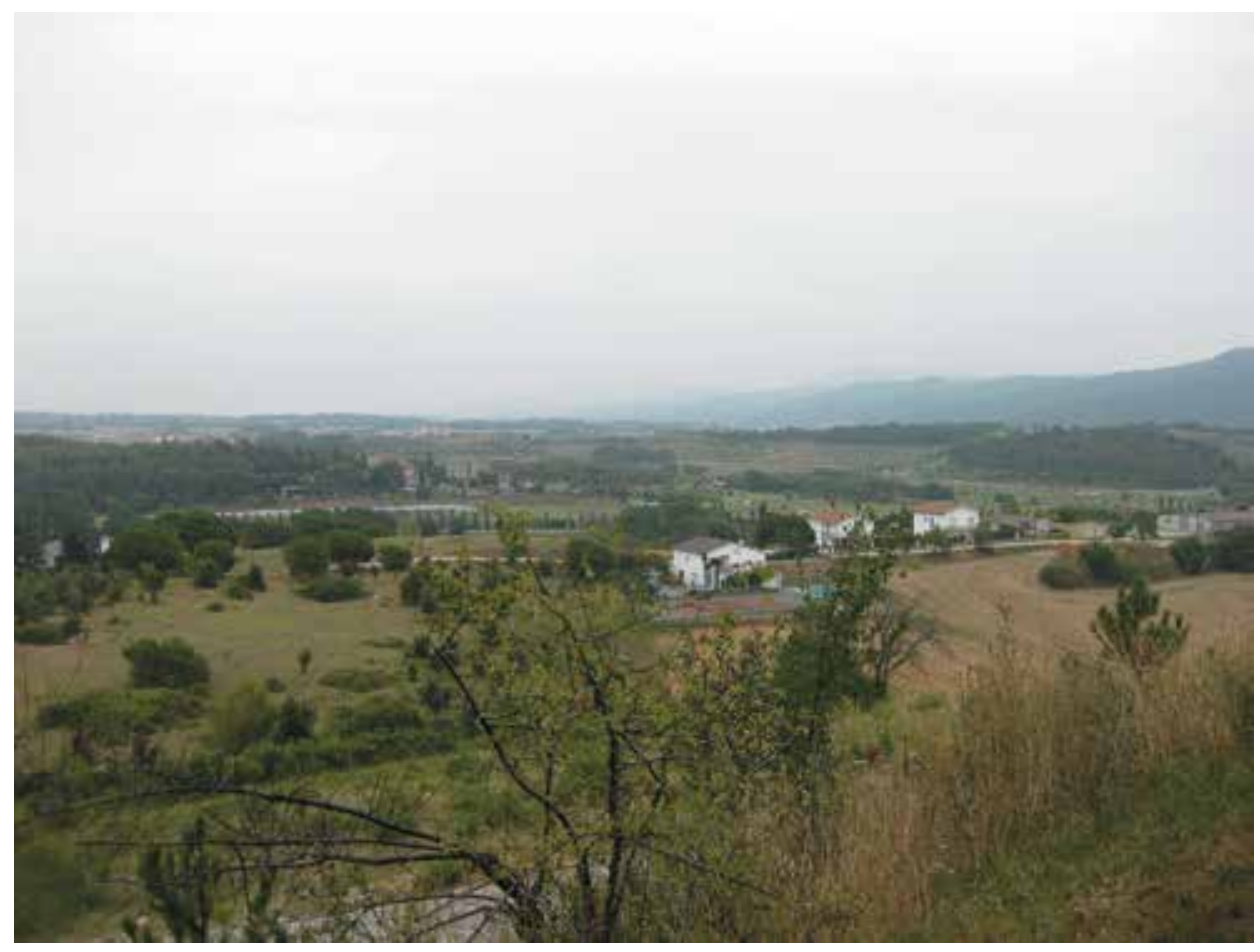

Figura I. Vista panorámica hacia el entorno circundante desde el conjunto escolar en la actualidad

Fuente: Amaya Martínez (201I)

bajo los conceptos de la modernidad arquitectónica durante la década de los sesenta, fue seleccionado por su innovadora propuesta. Su situación urbanística en un entorno carente de preexistencias arquitectónicas en el momento de su ejecución, pero con gran valor natural, implica que el conjunto en sí mismo ha caracterizado el lugar, y por lo tanto, la memoria del mismo.

Si bien el ejemplo acoge un uso educativo y residencial, y por lo tanto, unas especificaciones concretas, su formalización no dista del de otros edificios destinados a equipamientos, en los que organiza el edificio en torno a diferentes cuerpos articulados volumétricamente $y$ en los que se refleja el sistema estructural y constructivo escogido para dar una respuesta unitaria al conjunto. Por lo tanto, la permanencia y transformación de la Escuela-Residencia de Bell-lloc puede llegar a ilustrar una línea de actuación específica sobre las transformaciones de lo que podríamos definir como un "contenedor moderno".

Tras quedar en desuso, el conjunto educativo ha sido transformado recientemente, manteniendo su estado original. De esta forma, se ha convertido en la Sede Central de la Compañía Santa \& Cole y ha sido ampliado como Universidad Corporativa de Telefónica, tras la intervención de los arquitectos Batllé \& Roig.

Desvelar el proceso de transformación que ha experimentado la edificación tiene como objetivo evaluar los posibles criterios de intervención extrapolables a otros casos en los que su uso ha quedado obsoleto.

\section{LA FORMALIZACIÓN DE UNA ESCUELA MODERNA Y UNIVERSAL}

En el año 1964, Baldrich recibe el encargo de la Caja de Ahorros y Monte de Piedad de Barcelona y de la Diputación de Barcelona para construir una colonia veraniega. El encargo acabaría formalizándose en la construcción de una Escuela-residencia para enseñanza 
Figura 2a. Vista panorámica del complejo educativo en su acceso desde el oeste en la actualidad

Fuente: Amaya Martínez (2011)

Figura 2b. Vista del complejo educativo en la actualidad

Fuente: Amaya Martínez (2011)
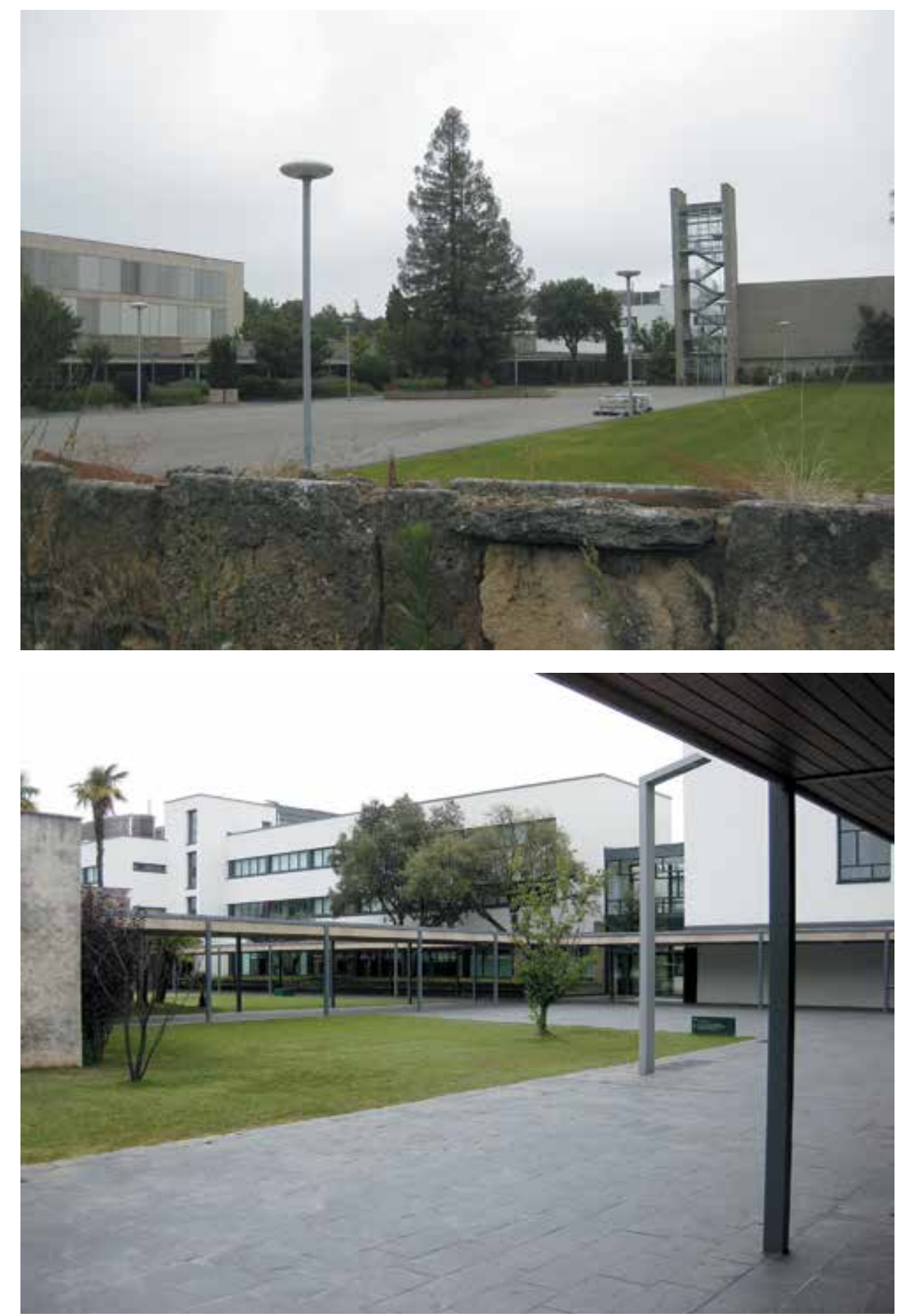

primaria y de bachillerato para $\mathrm{I} .200$ alumnos, que también serviría de residencia para 200 personas que incluían: personal docente, de servicios y los trabajadores de las obras de urbanización del conjunto. El lugar adquirido para la construcción del complejo se sitúa en el municipio de La Roca del Vallés, en la finca rural de Bell-lloc, con una superficie de 122 hectáreas (Armesto y Benito, 2008).

$4 \quad$ Debido a la escasa bibliografía existente sobre el edificio se destaca el artículo dedicado al conjunto escolar de Antonio Armesto y Mariona Benito en Quaderns, 2008. Los datos están siendo completados por la autora y la obra ha sido incluida en la tesis doctoral que desarrolla en la UPC sobre Arquitectura Escolar Moderna de Barcelona y Valencia. 
1. Aulario y residencia femenina

2. Administración

3. Aulario y residencia masculina

4. Comedor

5. Residencia de profesores

6. Residencia de profesoras

7. Iglesia

8. Sala de actos

9. Residencia de personal de servicio

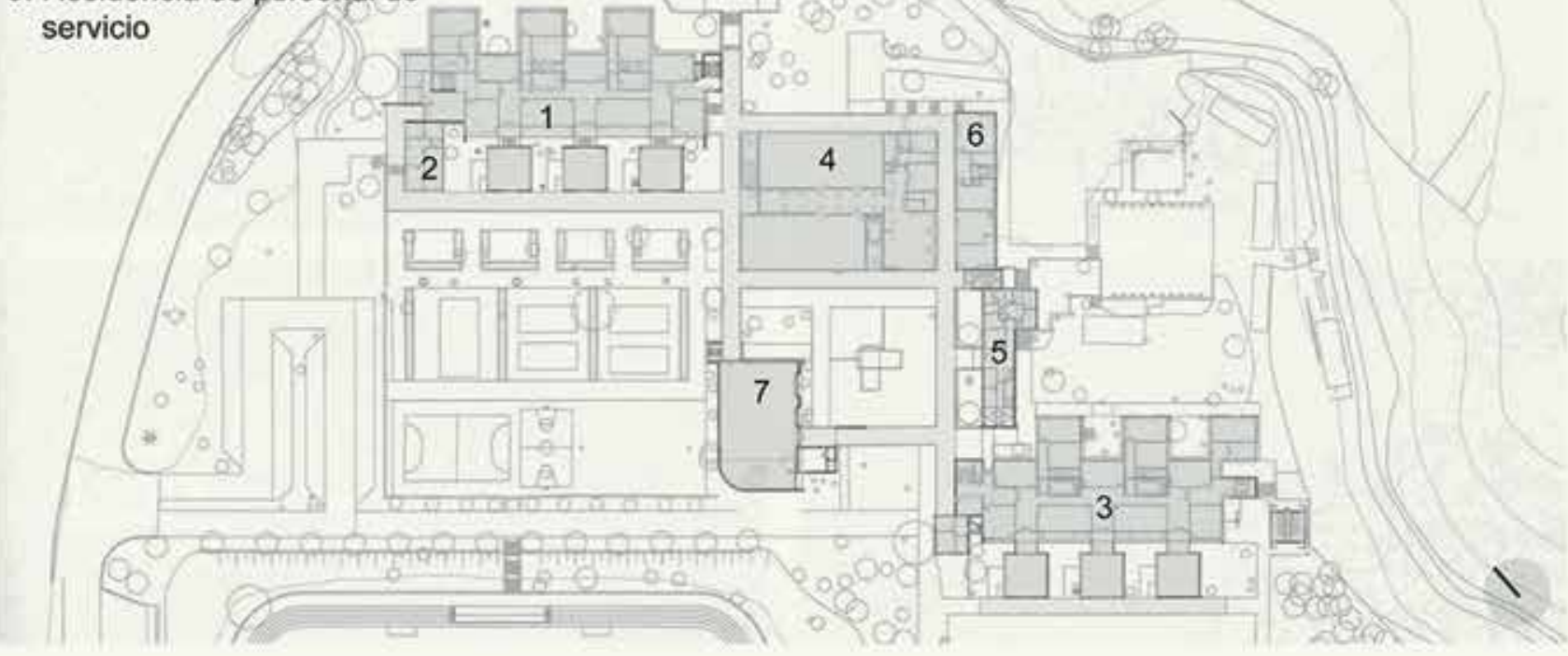


Figura 4. Vista actual del sur del edificio masculino como sede de la UCT (20II)

Fuente: Amaya Martínez (2011).

Figura 5. Vista actual de un patio de juegos, como área ajardinada y de exposición de los productos de Santa \& Cole (201I) Fuente: Amaya Martínez (2011).
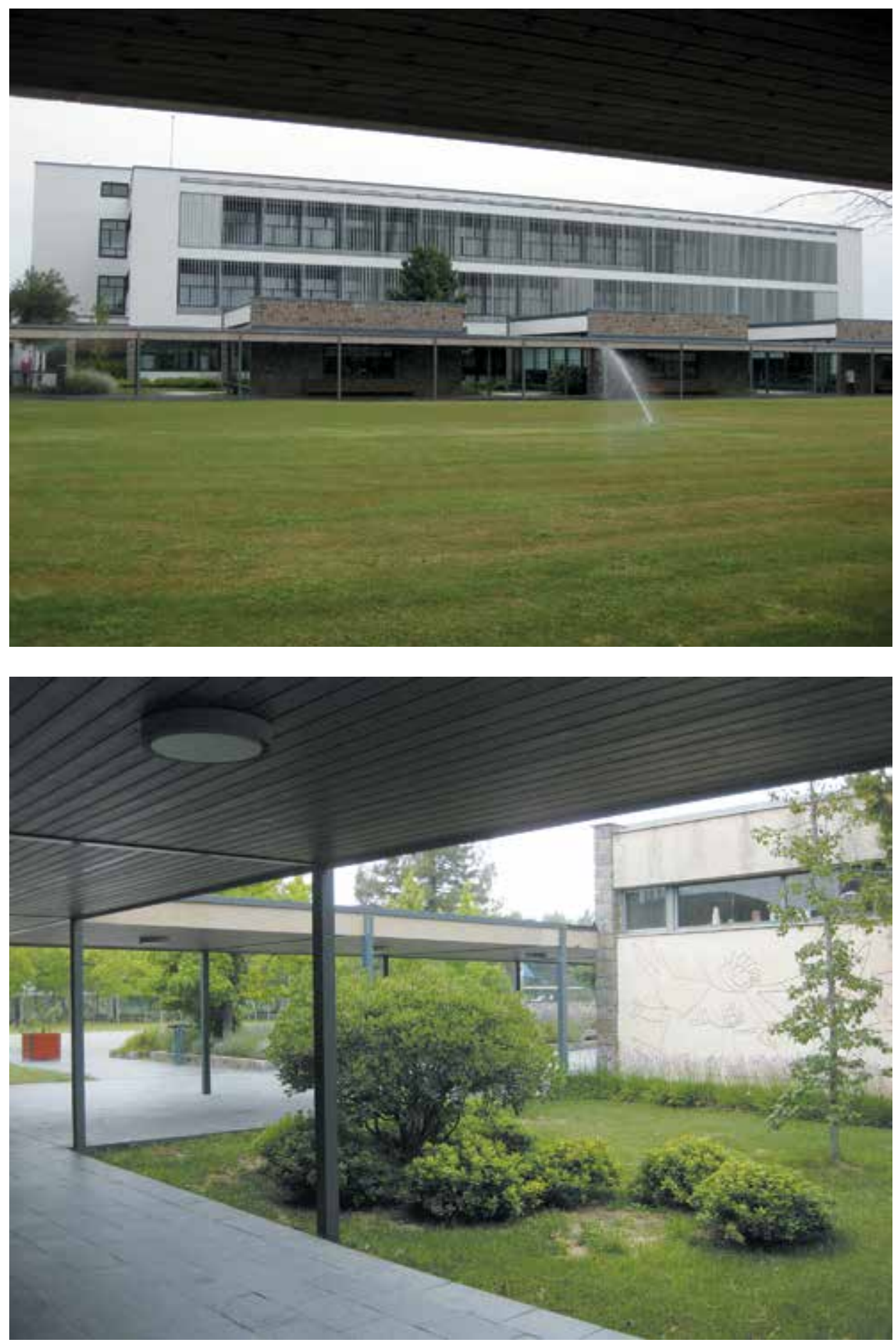

Las condiciones establecidas, las características del entorno, y la adscripción del autor a los valores de la modernidad arquitectónica en su línea más racionalista, dan como resultado una escuela fiel a los valores promulgados por la misma corriente. Una propuesta que invita a establecer una auténtica vinculación entre programa-lugar-forma.

Se decide situar el conjunto en la parte más elevada de la finca, una superficie que recibe previamente un considerable desmonte de 16 metros, para conseguir un espacio completamente plano, donde pueda construirse la edificación. 
Buscando la mejor orientación y a favor de una correcta ordenación del programa, se plantea una distribución en forma de $Z$, cuyos brazos extremos recogen, con una simetría espejada, las áreas destinadas a cada sexo. Cada uno de ellos, con tres niveles, resuelve las aulas en planta baja y la residencia en las superiores. El eje central de la $Z$ lo conforman el comedor, de un nivel, y la residencia de monjas y sacerdotes de tres niveles. El comedor se alinea al eje con el aulario femenino deslizándose hacia el sur, mientras que la residencia se resuelve en perpendicular al aulario masculino.

Del eje central de la $Z$ y transversalmente a él, penden dos volúmenes independientes, la capilla y el salón de acto. El primero domina el entorno con el campanario exento de la iglesia, el segundo se mimetiza con el lugar adaptándose a la pendiente del terreno y con el fin de resolver el graderío.

Resulta particularmente interesante la resolución dada a los edificios con uso mixto: aulario y residencia. En la planta baja se sitúan las aulas que, a modo de tapiz, sobresalen del volumen superior como unidades independientes, buscando una correcta iluminación y ventilación. La relación con el paisaje se produce de manera especial a través de los patios que, vinculados a cada aula, quedan abiertos en uno de sus frentes. Se produce así una extensión del espacio de docencia. Sobre la planta baja emerge el volumen que acoge la parte de residencias y dependencias anejas, ordenando el tapiz de aulas. Un prisma que parece levitar, con un lenguaje formal más racionalista, mostrando a través del telón que conforma la galería, una doble piel de brise-soleis y el plano retirado de acristalamientos.

En la ordenación urbanística toma una vital importancia la relación entre los volúmenes y el tratamiento dado a los espacios exteriores. Los caminos, cubiertos con un sistema de pérgolas, vinculan cada uno de los cuerpos aislados y dotan al conjunto de unidad a la vez que permite disponer de espacios de circulación independientes al paso a través de los edificios.

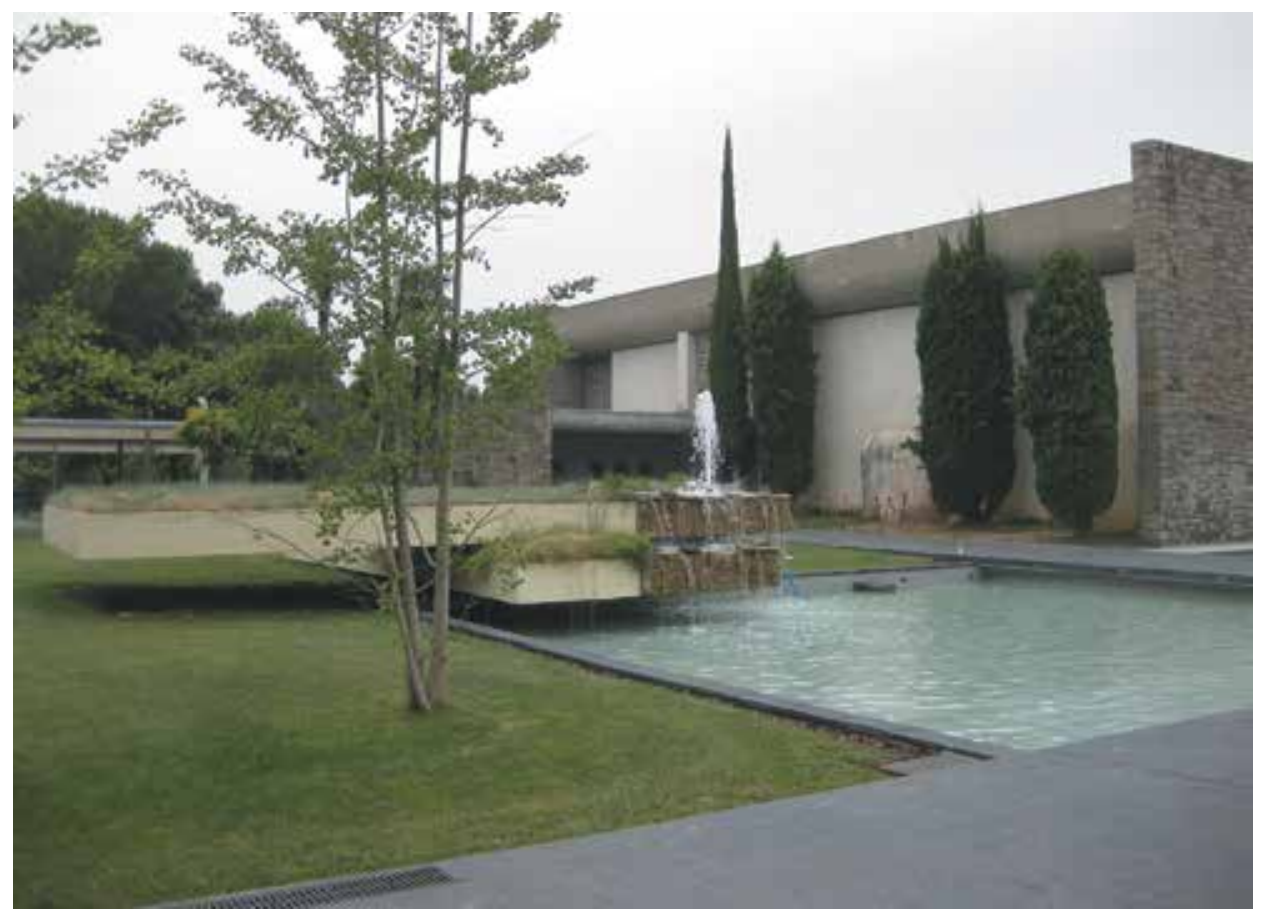

Se reconocen diversas áreas exteriores en el plano principal que quedan acotadas por la disposición de los diferentes volúmenes. El aparcamiento, situado en el acceso principal, al
Figura 6. El área ajardinada con la fuente y la iglesia al fondo (1969) Fuente: Amaya Martínez (201 I) 
Figura 7. El área ajardinada y la iglesia con el cuerpo exento del campanario Fuente: Amaya Martínez (201I). oeste; las áreas de juegos enfrentadas a cada uno de los espacios de docencia y el ágora y la plaza central, ubicada entre el comedor y la iglesia, un espacio cuidadosamente ajardinado en el que toma protagonismo la fuente con claras referencias a los planos de la Casa de la Cascada de Frank Lloyd Wright. Por debajo de la cota del plano principal y en dirección suroeste se sitúa la pista deportiva y la piscina. Todo el conjunto queda protegido del exterior por las masas arbóreas circundantes.

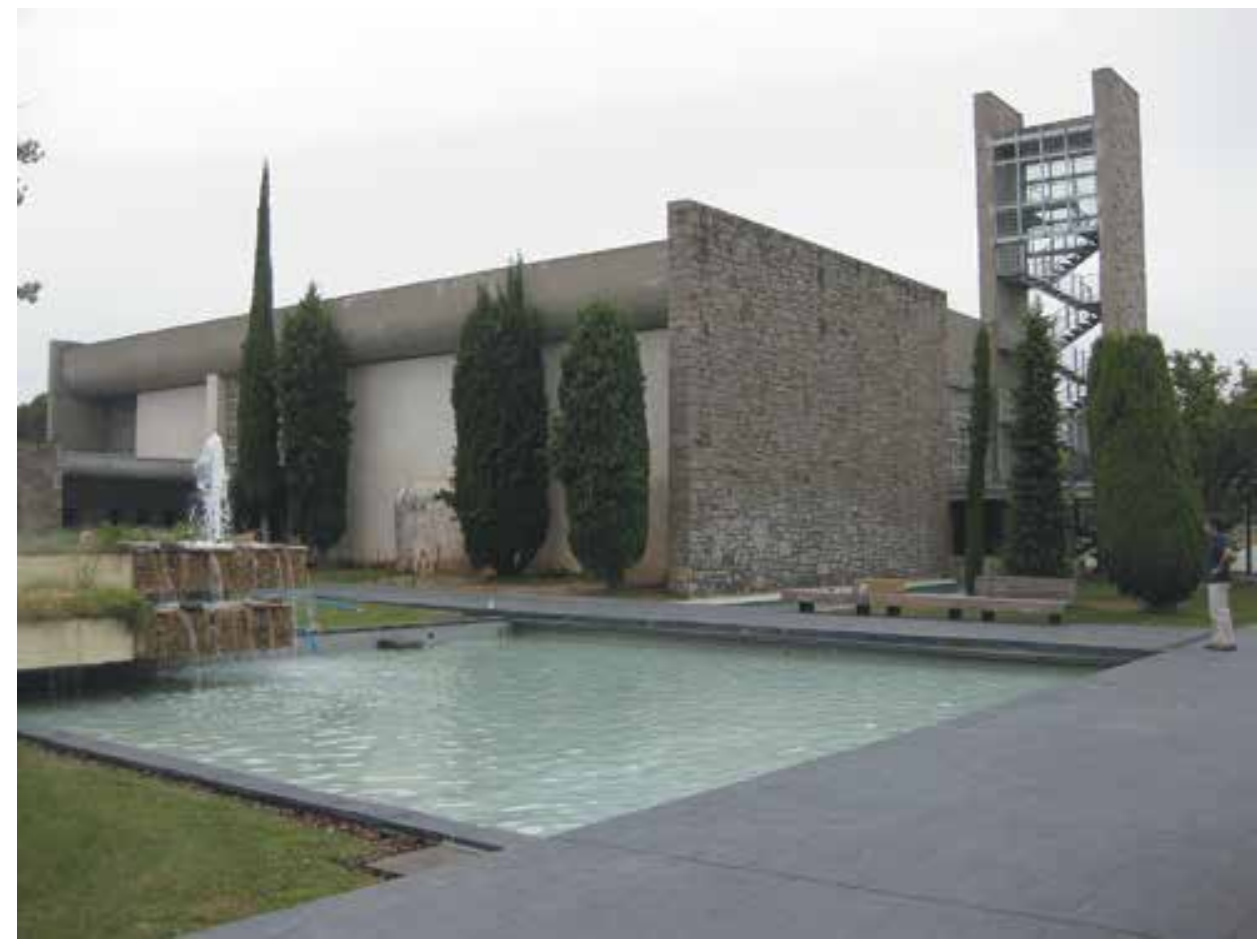

Además de la descripción anterior, Baldrich, quién también diseñó parte del mobiliario, se supo rodear de diferentes artistas para incorporar las artes como parte de la experiencia del aprendizaje. Así encontramos elementos artísticos en la iglesia como las esculturas y las puertas de J. M. Subirachs, las vidrieras de R. Casariada y los mosaicos exteriores sobre los muros de piedra de A. Martí que permiten identificar los diversos patios.

\section{INTERVENIR SOBRE LO EXISTENTE}

En el año 1994, se produce el cierre definitivo de la escuela-residencia debido a que, tras la fusión en 1992 de la Caja de Barcelona y la Caja de Pensiones para la Vejez y de Ahorros, se produce un cambio en la orientación de su política de ayudas hacia una vertiente más social. El Ayuntamiento de La Roca del Vallès, tras el cierre de la escuela, considerando los valores propios de la Finca de Belloch y del conjunto edificado, evitó su deterioro y destrucción, hecho que ha concretado con su inclusión en el Catálogo de Patrimonio del municipio.

En el año 2002, la finca es adquirida por Parc de Belloch S.L. filial de la empresa de diseño Santa \& Cole, con la intención de establecer sus oficinas centrales y fundar un parque tecnológico en un entorno privilegiado, próximo a la ciudad de Barcelona. El fotógrafo Jordi Cunill, a solicitud de la empresa de diseño, realiza en 2004 un reportaje fotográfico al conjunto. Sus imágenes muestran un edificio detenido en el tiempo y embebido por la naturaleza circundante. En su interior el mobiliario, abandonado súbitamente por sus usuarios, deja constancia de lo que allí acontecía. 

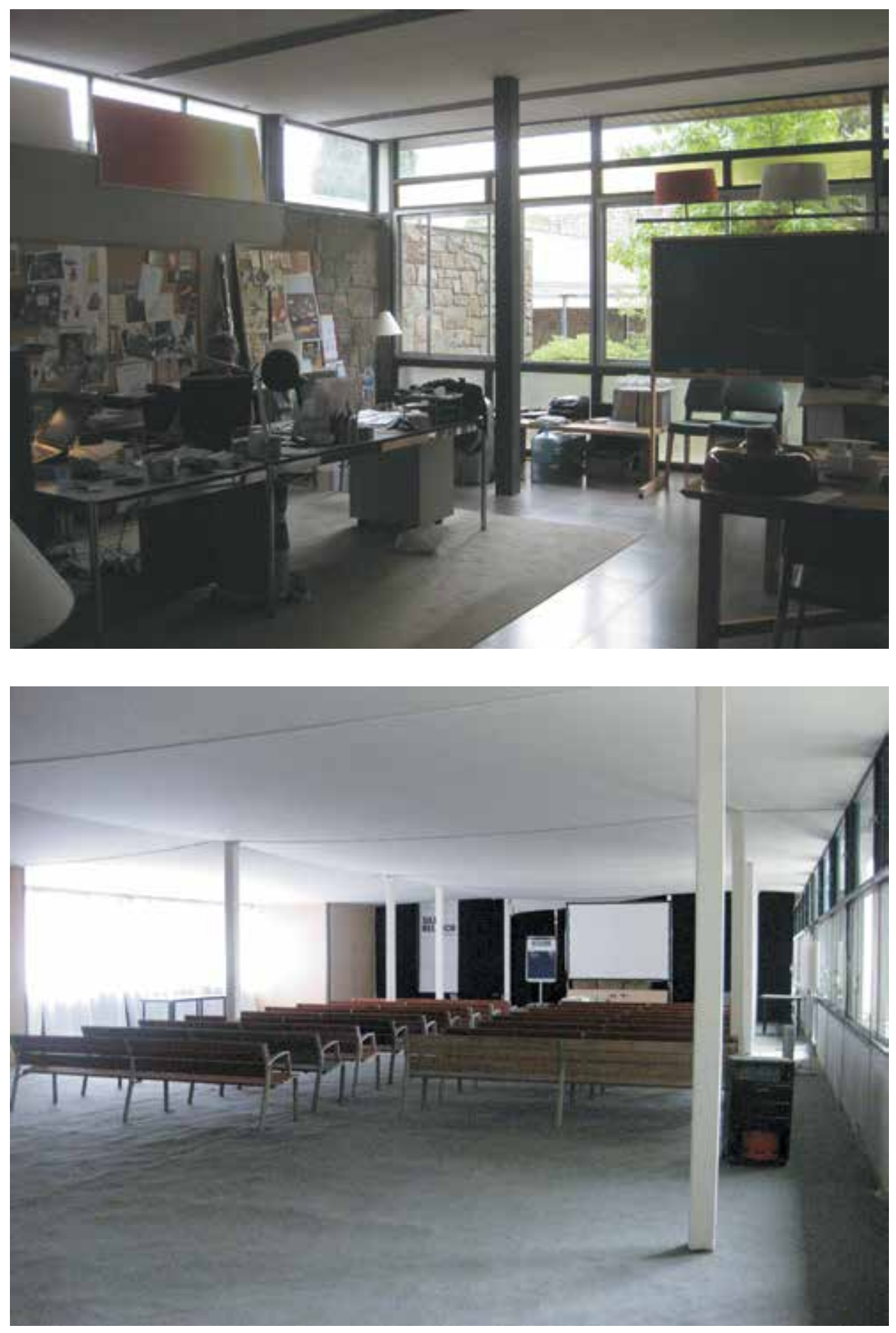

Parc de Belloch S.L. y Santa \& Cole se instalan definitivamente en el edificio destinado a las aulario-residencias, en la parte más próxima al aparcamiento. Las intervenciones que se realizan se limitan a modificaciones interiores para la adaptación a sus necesidades concretas. La planta baja es ocupada por la recepción y con los talleres de diseño ubicados en las aulas orientadas a sur, conformadas entre los patios. Las aulas internas son utilizadas como espacios de exposición de sus productos mientras las que vuelcan al norte se mantienen aún vacías, a espera de recibir nuevos usos. En la primera planta, se mantienen los paneles divisorios de los dormitorios y se adecúa una sala de presentaciones. Además, se realiza un
Figura 8. Interior de una de las aulas en la actualidad como áreas de diseño de Santa \& Cole. Se destaca la espacialidad a través de su altura y su relación con el exterior a través de las grandes superficies de cerramiento y los diferentes sistemas de ventilación Fuente: Amaya Martínez (2011)

Figura 9. Interior de una de las salas de residencia adaptada como sala de conferencias en la actualidad, en la que se han eliminado las mamparas de separación. Se destaca su espacialidad y la resolución de la fachada con el diseño de la carpintería para permitir una óptima ventilación y los brise-soleis Fuente: Amaya Martínez (20II). 
Figura 10. Vista aérea tras la reconversión, adecuación y ampliación de la Escuelaresidencia de Bell-lloc

Fuente: Batllè i Roig Arquitectes (2009), editada por la autora (2012) refuerzo estructural tras la eliminación de los tabiques longitudinales. Se eliminan los paneles divisorios interiores en la planta segunda y se distribuyen las oficinas. El diseño original ha permitido que se lleven a cabo estas variaciones sin que ello implique una merma de las calidades espaciales existentes en el edificio. El exterior se utiliza como espacio expositivo incorporando elementos de mobiliario urbano propio de la empresa.

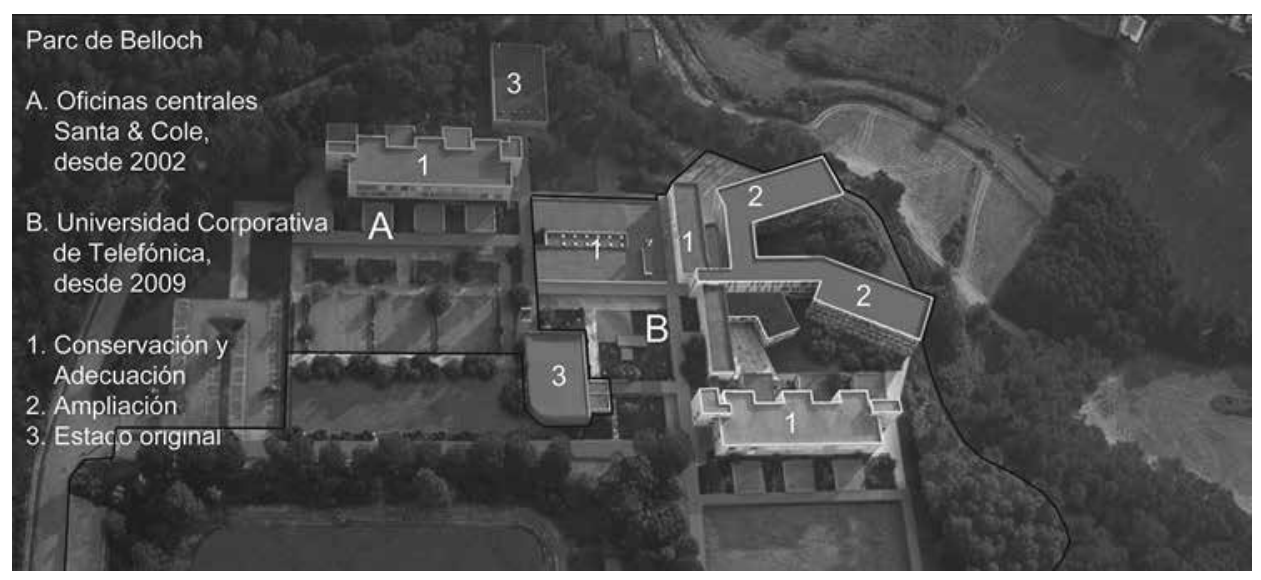

En 2006, los arquitectos Batlle i Roig reciben el encargo, por parte de Parc de Belloch S.L., de desarrollar una intervención de mayor envergadura: la adecuación en el resto de los edificios del Campus para la Universidad Corporativa de Telefónica (UCT). Debido a la amplitud del programa, que contaba con 180 habitaciones, 20 aulas para seminarios, restaurante, cafetería y salones, se decide intervenir y adecuar tres de los edificios existentes: el comedor, el aulario-residencia de alumnos y la residencia de profesores. Su extenso programa incluiría una ampliación destinada a acoger la nueva residencia y el acceso principal.

La intervención en el interior de los edificios existentes se realiza como un contenedor en el que se adecuan los espacios a las nuevas funciones; eliminando tabiquerías e incorporando nuevos elementos como escaleras y ascensores para cumplir con la normativa. Las fachadas son rehabilitadas y se sustituyen las carpinterías y las superficies acristaladas para cumplir con las normas de aislamiento. Así, se decide mantener su despiece original para no alterar la imagen del conjunto.

La ampliación da origen a una nueva pieza que recoge la ortogonalidad de la trama existente y se quiebra, buscando la mejor orientación y vistas hacia el paisaje. En estos dos nuevos brazos se ubican los dormitorios. Su materialidad, con grandes superficies acristaladas, y un juego de travesaños de madera, entra en un claro contraste con la alternancia de huecos y muros ciegos de estuco o piedra de los edificios originales.

La intervención arquitectónica se completa con la adecuación de los espacios exteriores: el ajardinamiento y la incorporación de nuevas pérgolas que terminan de unir los nuevos recorridos peatonales, a tal punto se reproducen los originales que no es posible distinguir entre unos y otros.

A través de los conceptos "conservación”, “adecuación” y "ampliación” se ha definido el proceso de transformación de un conjunto educativo-residencial en un parque tecnológico. Una transformación en la que ha primado el respeto por lo preexistente, tanto por sus valores patrimoniales como por un esfuerzo en mantener aquello que todavía es útil, en pro de la sostenibilidad, y sin olvidar los valores actuales del momento en el que se interviene. 


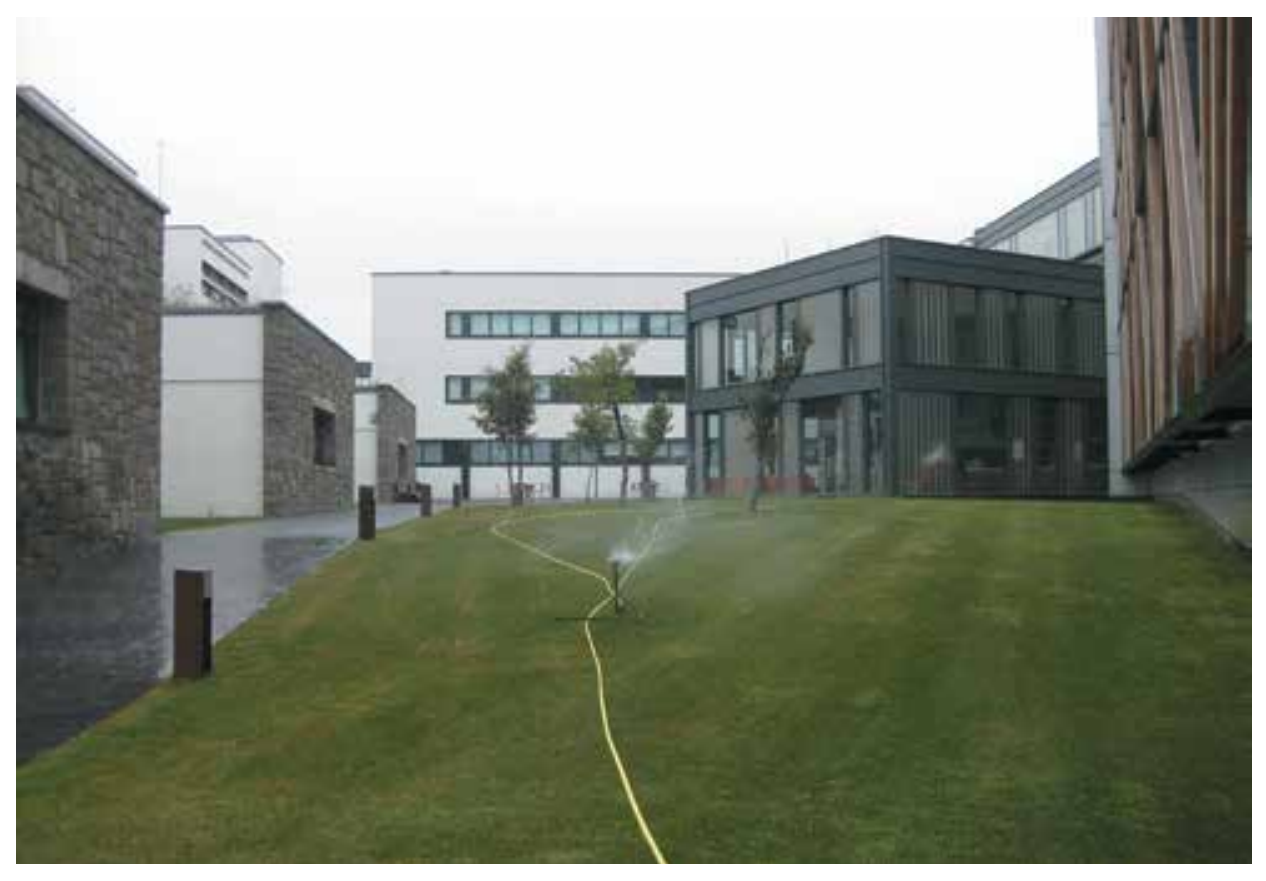

Figura II. Fusión entre lo "Antiguo" y lo

"Nuevo" tras la intervención

Fuente: Amaya Martínez (2011).

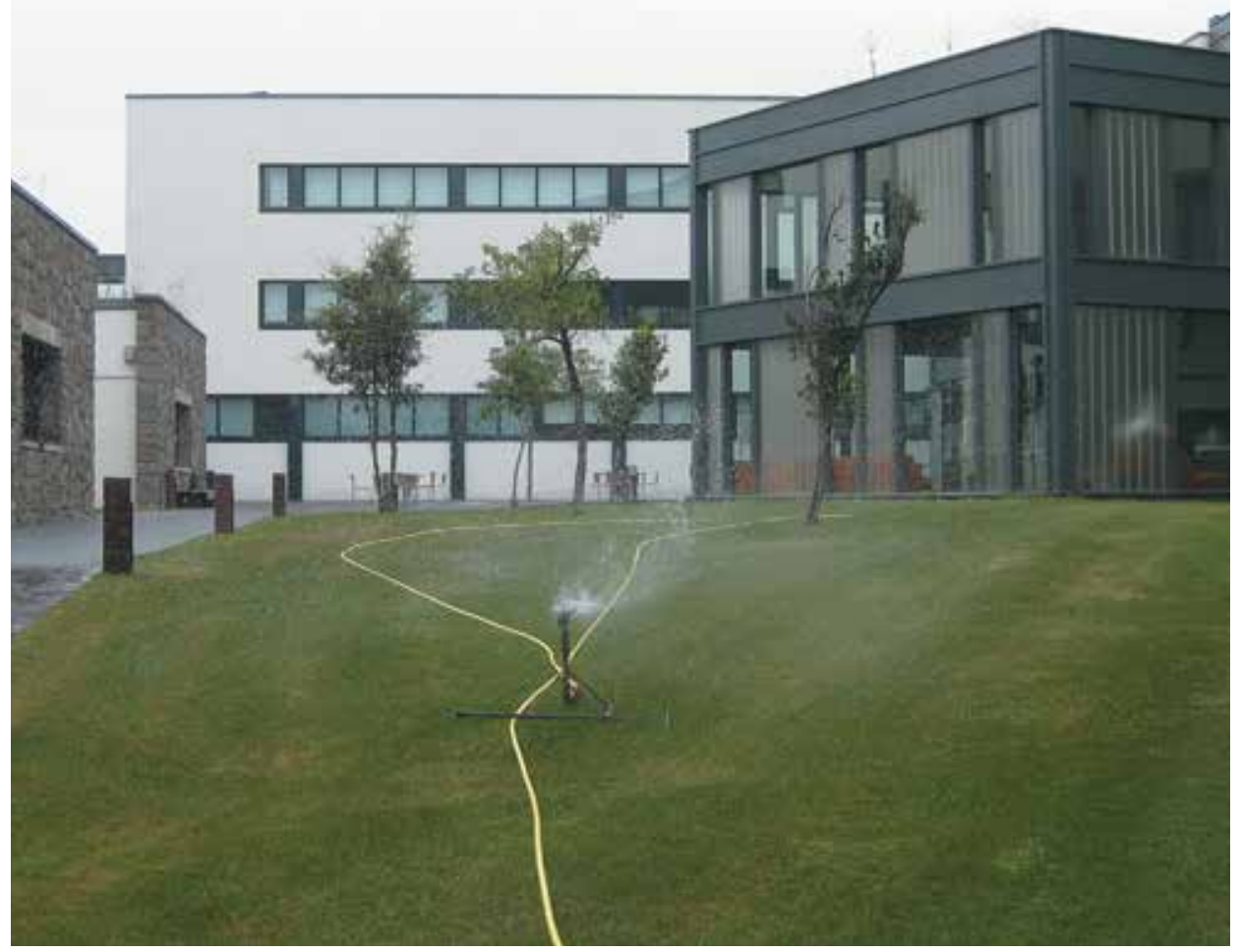

M 131 REVISTA M VOL. 10 No.2. JULIO-DICIEMBRE 2013 • FACULTAD DE ARQUITECTURA • UNIVERSIDAD SANTO TOMÁS COLOMBIA 
Figuras 12 y 13 . Fotografía aérea antes

(2007) y después de la intervención (2009)

Fuente: Google Earth.
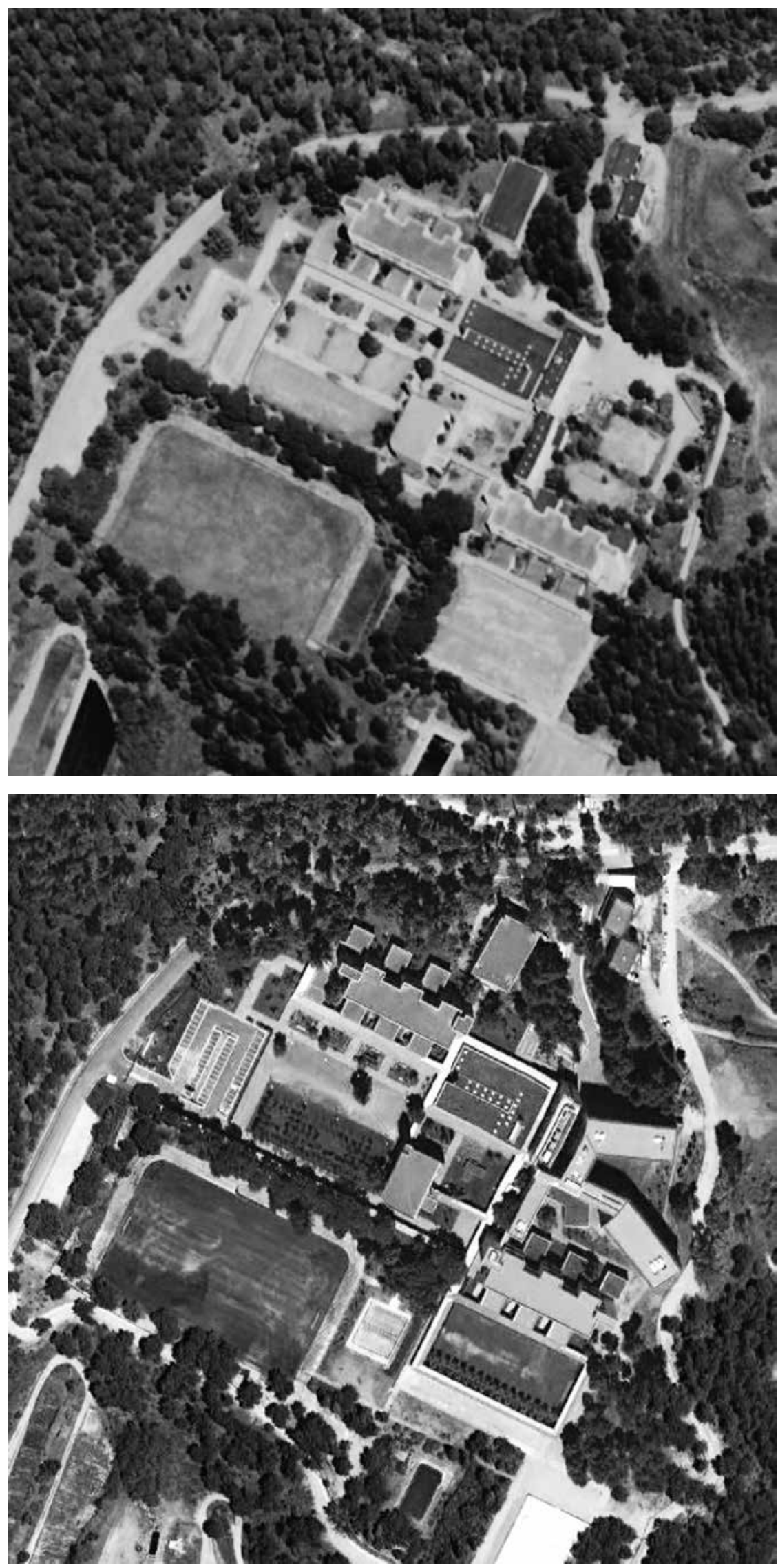


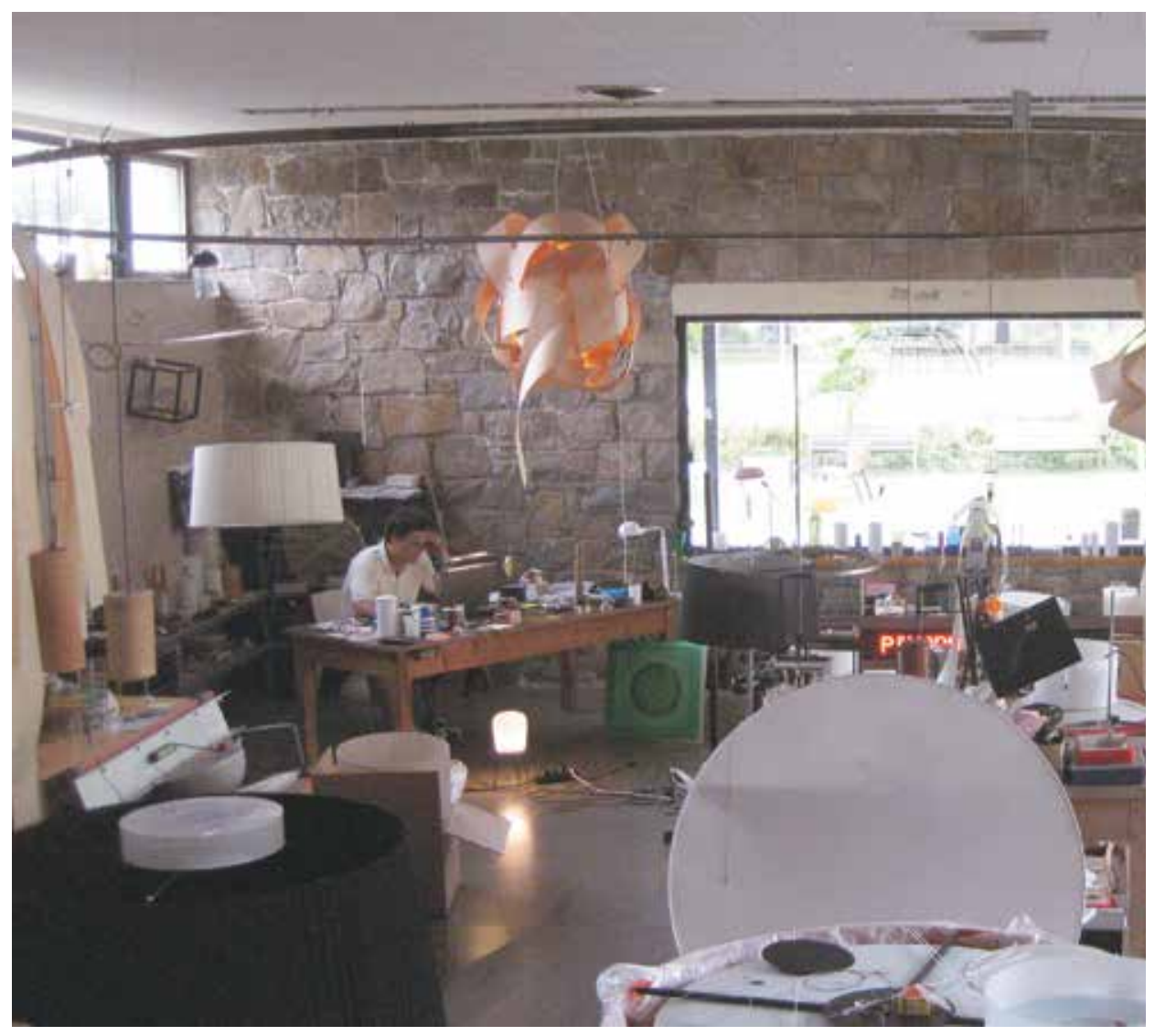

Figura 14. Aula del bloque femenino de la Escuela-Residencia de Bell-lloc transformada en taller de diseño de lámparas de Santa \& Cole

Fuente: Amaya Martínez (2011).

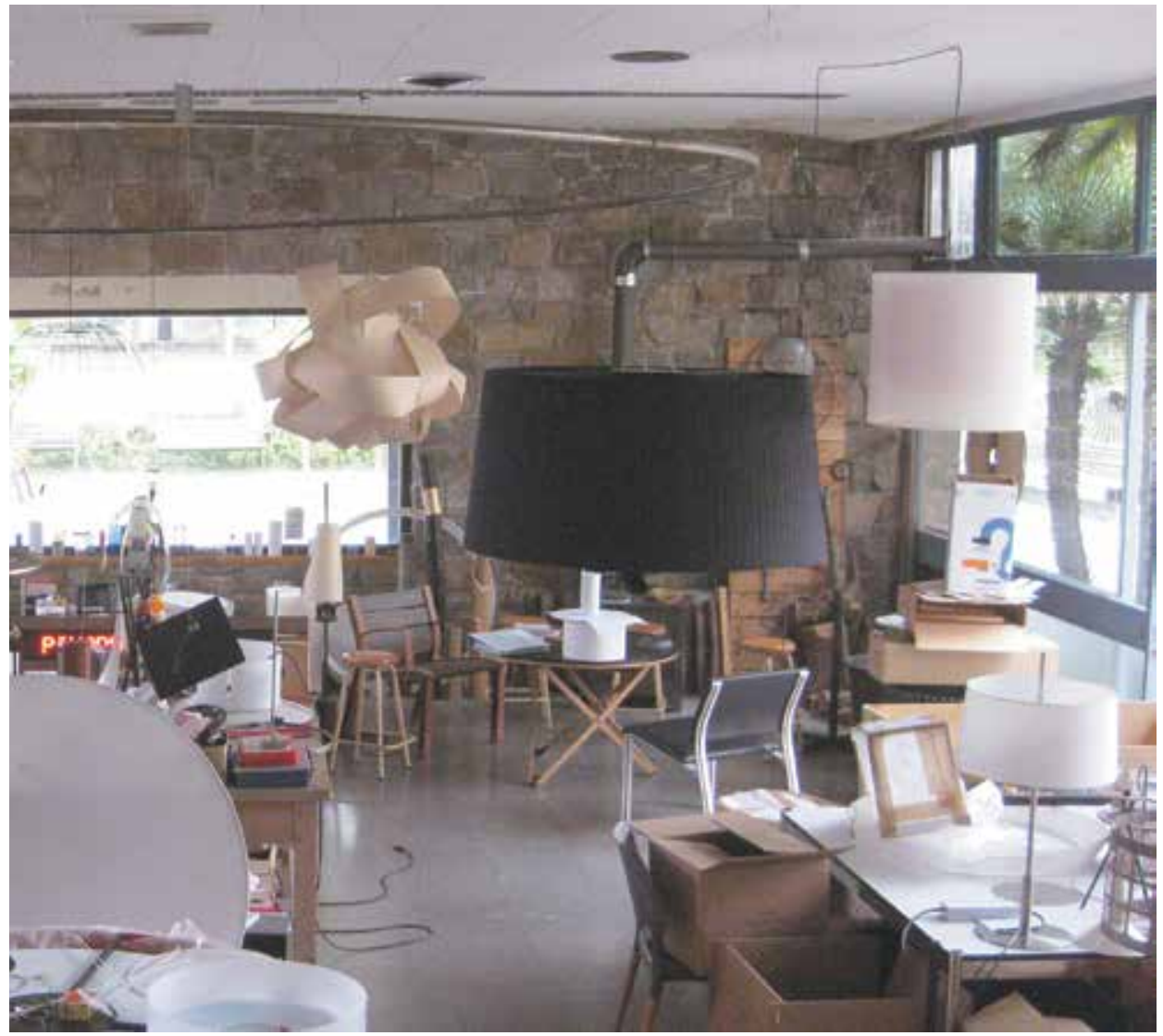

M 133 REVISTA M VOL. 10 No.2. JULIO-DICIEMBRE 2013 • FACULTAD DE ARQUITECTURA • UNIVERSIDAD SANTO TOMÁS COLOMBIA 
El conjunto escolar es capaz de abstraerse de su propia definición para ser considerado en términos generales como un "contenedor moderno", un contenedor cuya función primigenia podría sucumbir, pero no su orden, permitiendo la reutilización de sus espacios.

Batllè \& Roig manifiestan un pretendido interés por "respetar, conservar y recuperar aquello que caracteriza la construcción primigenia, pero introduciendo adaptaciones para lograr una simbiosis entre lo restaurado -lo antiguo- y lo ampliado -lo nuevo-" (A.G., Margelef, responsable del proyecto, entrevista realizada el 26 de octubre de $201 \mathrm{I}$ ).

El conjunto, en uso desde 2009, se encuentra aún hoy en proceso de recuperación de diferentes áreas de la sede de Santa \& Cole y de los edificios de Sala de actos e Iglesia.

\section{CONCLUSIONES}

El conjunto escolar comienza siendo una escuela-residencia $y$, tras un periodo en desuso, se adapta y transforma para convertirse en un parque empresarial, Parc de Belloch, sin perder su identidad. Este hecho ha sido fundamentalmente gracias a los valores formales intrínsecos del conjunto escolar original. Sin embargo, hay que reconocer la apuesta y el esfuerzo de los promotores privados por recuperar este patrimonio reciente, pero no por ello menos importante. La empresa Santa \& Cole, ha fomentando su transformación y reconversión en lo que en la actualidad, una apuesta por un nuevo parque del conocimiento y del diseño. En el que, además, se ha instaurado la nueva sede de la Universidad Corporativa de Telefónica que ha resuelto sus necesidades bajo el proyecto de adecuación y ampliación de los arquitectos Batllé \& Roig (2009).

El éxito de la reconversión se debe a la confluencia de diversos factores:

I. El buen estado de conservación del edificio original. Su estado había quedado asegurado tanto por la calidad de los materiales utilizados como por la correcta ejecución con la que se había llevado a cabo. En este sentido, y pese al deterioro lógico debido al abandono y el paso del tiempo, en la intervención ha primado la idea de recastar y la restaurar elementos originales como las carpinterías de madera, las pérgolas o los elementos artísticos.

2. La calidad del diseño de los espacios originales interiores y exteriores. Aspectos como las dimensiones polivalentes de pasillos, la óptima resolución de los elementos de comunicación vertical, la distribución y las óptimas proporciones de las aulas. A nivel exterior destacan el cuidado del diseño, la zonificación de los amplios espacios, los ajardinamientos y la inclusión de elementos como la fuente o los recorridos peatonales bajo las pérgolas.

3. La delicada inserción de lo nuevo frente a lo viejo. Se produce un diálogo respetuoso con el edificio original, pero atendiendo al momento actual. Hecho que queda manifiesto en los nuevos volúmenes construidos que no alteran la imagen del edificio original. La materialidad, especialmente en el exterior es rehabilitada y se mantienen aspectos relevantes para la imagen del conjunto como los despieces de las carpinterías. 
La reconversión del conjunto así como la reutilización y restauración de números elementos constructivos y materiales aseguran trabajar bajo los conceptos de sostenibilidad en un momento en el que los recursos son cada vez más limitados y el entorno se encuentra saturado de estructuras existentes capaces de albergar nuevos usos. Con todo ello, el edificio escolar $y$ sus leyes formales demuestran una gran versatilidad, ofreciendo un escenario donde poder trabajar a partir de conceptos $R E$ para así persistir a través del cambio.

More over, built environment have lives of their own: they grow, renew themselves, and endure for millennia. Conservation may serve to freeze works of art in time, resisting's time effects. But the living environment can persist only through change and adaptation (Habraken, 1998).

\section{REFERENCIAS}

AA.W. (1964). Carta de Venecia. Carta Internacional sobre la Conservación y la Restauración de los Monumentos y los Sitios. Segundo Congreso de Arquitectos y Técnicos de Monumentos Históricos en Venecia, 25 al 31 de Mayo de 1964.

AA.W. (20II). Madrid Document for Twentieh-Century Architectural Heritage. Conferencia Científica Internacional sobre los criterios de intervención en el Patrimonio Arquitectónico del Siglo XX.

Armesto, A., Benito, M. (2008). Escuela-residencia de Bell-lloc (1964-1968). Arquitecto: Manuel Baldrich Tibau. En: Quaderns d'Arquitectura i Urbanisme, 257. 104-I I3.

Batllé \& Roig Arquitectes (2009). Universidad Corporativa de Telefónica en el Parc de Belloch en La Roca Del Vallés, Barcelona. En:Temas de Arquitectura, 9, I38-I 55.

Habraken, N. J. (1998). The structure of the Ordinary. Form and Control in the Building Environment. Cambridge and London: MIT Press.

Montaner, J. M. (20I I). Una nueva sociedad: hacia la rehabilitación. En: Visions de l'Escola Tècnica Superior d'Arquitectura de Barcelona, 9, 6-I3. 